\title{
Reaching a consensus on the mechanism of dynamin?
}

\section{Aurélien Roux}

Address: Biochemistry Department, University of Geneva, CH-1211 Geneva, Switzerland and Swiss National Centre for Competence in Research Programme Chemical Biology, CH-1211 Geneva, Switzerland

Email: aurelien.roux@unige.ch

Fl000Prime Reports 2014, 6:86 (doi:10.12703/P6-86)

All Fl000Prime Reports articles are distributed under the terms of the Creative Commons Attribution-Non Commercial License (http://creativecommons.org/licenses/by-nc/3.0/legalcode), which permits non-commercial use, distribution, and reproduction in any medium, provided the original work is properly cited.

The electronic version of this article is the complete one and can be found at: http://fl000.com/prime/reports/b/6/86

\begin{abstract}
Among the proteins involved in lipid membrane remodeling in intracellular traffic, dynamin has been the focus of many studies, as it was the first protein shown to be mechanistically involved in membrane fission: the reaction by which a vesicle neck can be severed to release a free vesicle. After almost 25 years of research, a wide variety of data from various techniques has been acquired on the mechanism by which dynamin breaks membranes. However, the literature may sometimes sound confusing, and the primary goal of this review will be to provide a stepping stone towards a potential consensus on how dynamin may work. I will then discuss the most recent findings in light of previous work, and the future possible lines of research in the field of dynamin.
\end{abstract}

\section{Introduction}

One of the essential reactions required for intracellular membrane traffic is fission: a membrane compartment that is separated from another one by the breakage of the thin neck of membrane connecting them. It is the reverse reaction to fusion, which is usually mediated by soluble N-ethylmaleimide-sensitive factor attachment protein receptor (SNARE) proteins. The first protein ever implicated in the fission reaction was dynamin in the early 1990s [1]. Its higher-order structure, a helical collar at the neck of clathrin-coated pits (CCPs), immediately suggested a mechanism: if the dynamin helix constricts, it could sever the membrane by squeezing it to an infinitely small radius. The last 15 years have seen an accumulation of data supporting this view, but with unexpected twists to this initial model. The goal of this review is to describe the findings that could be the basis for a widely accepted mechanism of dynamin function. In particular, the constrictive ability of dynamin, which is generally accepted. In the second part of the review, I will detail the latest ideas about how this constriction could lead to fission and describe the pros and cons of each hypothesis. Finally, I will try to address the emerging areas of dynamin research, in particular the function of the numerous partners of dynamin and the coupling between constriction and the GTP cycle.
The facts: dynamin, a constriction enzyme

Dynamin is $100 \mathrm{kDa}$ GTPase that exists in tetrameric [1] or dimeric [2] form in solution. However, in the absence of GTP, it can oligomerize into a helical polymer of about $50 \mathrm{~nm}$ in width with a $20 \mathrm{~nm}$ inner diameter $[3,4]$. Dynamin was the first protein shown to possess membrane tubulation activity when mixed with liposomes $[5,6]$. Because the characteristic helical pattern seen on the tubes matched the dimensions of the dynamin oligomer formed in the absence of membrane, it was proposed that dynamin deforms the membrane by forming a scaffold. However, at low concentrations of dynamin, a competition between the polymerization energy of dynamin and the energy required to deform the membrane leads to the membrane curvaturedependent nucleation of dynamin [7]. Dynamin could thus be recruited to the neck of growing clathrin buds, at sites where curvature is built up by clathrin polymerization to the point where curvature is sufficient to promote dynamin nucleation.

In the presence of GMP-PCP [8,9] (a non-hydrolysable analog of GTP), these tubes can constrict to an outer diameter of approximately $40 \mathrm{~nm}$ [5,8-11], leaving a membrane tube inside the dynamin coat with a lumen that has a $3.5 \mathrm{~nm}$ radius. This important piece of information 
strongly supports the constriction model. Indeed, the recently published crystal structure of dynamin $[2,12]$, combined with the three-dimensional (3D) helical reconstruction of the dynamin-coated tubules obtained from cryo-electron microscopy (cryo-EM) data, confirmed that GTP hydrolysis induces a conformational change at the dimer level and triggers constriction [9]. The GTPase domains from adjacent turns of the dynamin helix interact, creating bonds between the turns [13]. Also, the number of dimers per helical turn was reduced from 14 to 13 [8], suggesting that part of the constriction was also coming from the torsion of the helix [8]. Indeed, by using the live imaging of dynamin-coated tubules undergoing GTP treatment, a rotation of beads attached to the dynamin coat was observed [14], confirming that the constriction of the dynamin helix was achieved by twisting. Moreover, this torque, applied by the dynamin helix onto the membrane while constricting, is sufficiently large to constrict the membrane.

Thus, the ability of the dynamin polymer to constrict upon GTP hydrolysis is supported by structural data, biophysical data, and biochemical data. These findings are the basis of a consensus for the dynamin mechanism. However, there is still one major open question about the constriction of dynamin: how much can it constrict the membrane?

The first cryo-EM 3D reconstruction images, which showed a lumen with a $3.5 \mathrm{~nm}$ radius, were obtained in the presence of non-hydrolyzable analogs of GTP $[8,9,15]$ that do not trigger fission in most in vitro assays (see below). Thus, it was believed that the constriction seen on these images was not the maximal constriction of dynamin. The Hinshaw group recently obtained a much more constricted 3D map of the dynamin helix [16]. The group used the K44A mutant of dynamin that has low GTPase activity in the presence of GTP. In this map, the radius of the lumen goes down to $1.9 \mathrm{~nm}$, which is close to but still above the theoretical value at which membranes spontaneously break [17]. Therefore, while these results confirm that dynamin can constrict membranes, this constriction apparently does not reach the hemi-fission state (where only a cylindrical micelle of membrane is left inside the dynamin coat). This tends to support the idea that dynamin does not break the membrane by pure constrictive forces alone (see below). There may be two, non-exclusive reasons for the inability of dynamin to reach the hemi-fission state by constriction. Firstly, it is probable that the energy required to reach hemi-fission is unfeasible, especially considering that the dynamin oligomer already requires a huge torque to constrict to $1.9 \mathrm{~nm}$ [18]. The second possibility could be that it is structurally very difficult to build a machine that can induce a conformational change from $10 \mathrm{~nm}$ to $0 \mathrm{~nm}$ by constriction.
The value of the minimal constriction radius may seem to be a detail, but it is linked to a much more essential question: how does constriction trigger membrane fission? This question has been the subject of close scrutiny for many years, but recent findings have revised our thinking about the mechanism by which dynamin could trigger membrane fission.

\section{The current discussion: how does constriction lead to fission?}

Many models have been proposed to explain how dynamin-mediated membrane fission occurs. However, since the bulk of the experimental data support a constriction-based mechanism, the debate has focused on how the constriction of the dynamin ring would lead to fission. There have been three recent explanations of how membranes would be broken by constriction. Firstly, if the membrane is constricted enough, fission can occur at the edge of the dynamin coat spontaneously through membrane fluctuations (Brownian motion). This process is activated by increased membrane tension. Secondly, highly constricted membranes could spontaneously undergo fission when the dynamin coat disassembles upon GTP hydrolysis. Thirdly, the final constriction leading to hemi-fission is linked to a local bending of the membrane induced by the rotation of the pleckstrin homology $(\mathrm{PH})$ domains present in the dynamin monomers. In the following section, I review the experimental work supporting or in opposition to each hypothesis and discuss how to test them in a more quantitative way.

\section{Membrane fission is tension dependent}

In addition to the cryo-EM data discussed above, other data suggesting that dynamin constriction was not sufficient for fission were produced by studies testing the constriction model. In particular, the striking finding from the Hinshaw group that dynamin tubules snap into membrane beads when attached to the EM grid prior to GTP treatment [5] (whereas they would simply constrict when treated in solution [10]) showed that constriction was not sufficient for fission but that attachment to a substrate was also required. Indeed, when followed by live imaging, dynamin tubules would snap only when attached to the substrate, as a result of the tension generated by the torsion of the helix [14]. Thus, tension was required for dynamin-mediated membrane fission, which required actin polymerization $[19,20]$ in vivo, the main membrane tension regulator, and can generate pulling forces on endocytic structures $[20,21]$.

It became clear that the mechanics of the membrane could strongly impact on the dynamin-mediated fission reaction. In an in vitro assay [18], membrane tension and rigidity were recently shown to strongly impact the kinetics of the fission reaction. The kinetics were predicted 
by a theoretical model in which membrane elastic energy was increased at the edge of the constricted dynamin coat, resulting from the steep change in curvature between the constricted part of the membrane (dynamin-coated) and the non-constricted part of the membrane (uncoated). This model predicted that fission would preferentially occur at the edge of the dynamin coat, where the elastic energy of the membrane is the highest. It also predicted that increased membrane tension would reduce the time it takes for fission. In reconstitution assays, where membrane composition could be controlled, fission actually occurred at the edge of the dynamin coat and was faster if membrane tension was increased [18].

In short, this model explains the contribution of constriction and of membrane tension in membrane fission: constriction is required to bring the membrane close to a hemi-fission state. Then fission would be spontaneously triggered by thermal fluctuations of the membrane at the edge of the dynamin coat where the membrane is free to fluctuate, but is still highly constricted. So dynamin puts the membrane in a configuration where thermal energy can activate fission. The price to pay for not having reached the hemi-fission intermediate state is the requirement to wait for spontaneous fission: it is highly stochastic and takes, on average, a few seconds. In further support of this model, both in vitro and in vivo, time for fission was found to be highly stochastic and on average about 5-10 seconds $[18,22,23]$.

However, there is a limitation to this model. The time it takes for fission is dependent on $e^{1 / R^{2}}$ where $R$ is the constriction radius of dynamin. Thus, a small change in the constriction radius can lead to changes of several orders of magnitude in the fission time, making it critical to know the constriction radius of dynamin to test this model. For a constriction radius of the membrane below $5 \mathrm{~nm}$, as shown by the Hinshaw group $[8,15,16]$, fission is expected to occur within a few seconds to a few tens of seconds. A recent striking finding has been presented in support of this model [24]. Polyunsaturated acyl chains reduced the effective bending rigidity of the membrane, dramatically reducing the energy required for constriction. This probably accounts for the very fast dynamindependent fission reaction observed in neurons $[25,26]$ as the plasma membrane of neurons is particularly rich in polyunsaturated acyl chains.

\section{Disassembly of dynamin}

Dynamin in cells disassembles after fission, and disassembly is thought to be primarily caused by the hydrolysis of phosphatidylinositol 4,5-bisphosphate $\left(\mathrm{PIP}_{2}\right)$ by synaptojanin [27-32]. In vitro, dynamin disassembly was first shown to be GTP-dependent by Hinshaw and colleagues [4].
Moreover, the soluble fraction of dynamin increased in the supernatant of liposomes that had been decorated by dynamin and treated with GTP [5]. Also, the light-scattering signal of dynamin-decorated liposomes first shrank within a few seconds of treatment with GTP, but later increased, possibly reflecting a disassembly process $[5,10,33]$. Indeed, by EM, wildtype (WT) dynamin was found to disassemble after a few minutes, a similar time to that observed in the light-scattering experiments. Using supported bilayers with excess membrane reservoir (SUPER) templates, a novel tool for both imaging and biochemistry, Pucadyil and coworkers [33] showed that there is a time correlation between fission and disassembly and that long membrane tubes fully coated with dynamin (created by adding dynamin first, and then GTP) are less potent for fission than small assemblies of dynamin (where dynamin and GTP are co-injected). They proposed a mechanism that coordinates the fission with the disassembly: when the highly constricted membrane is still coated by dynamin, it cannot break. The rapid disassembly of dynamin could, therefore, lead to the destabilization of the membrane that would fuse with itself and break.

Further support for this model came from a technically challenging in vitro assay [23]: dynamin was assembled onto tubes extracted from black lipid membranes, and the radius of the tube was followed by monitoring the electrical current of the luminal ions [23]. Surprisingly, the authors observed an increase in conductance when GTP was added to the tube, suggesting an initial widening rather than a constriction induced by dynamin. Subsequent to this, a rapid dwindling of the conductance was measured, signaling tube scission. The authors concluded from the increase of conductance prior to fission that dynamin was depolymerizing, widening the tube.

Even though this is a seductive mechanism for membrane fission as it is consistent with the rapid disappearance of dynamin at endocytic buds seen during fission in vivo $[22,34]$, the induction of membrane fission by the depolymerization of dynamin is a priori incompatible with the known mechanics of lipid membranes. Depolymerization of dynamin (by releasing the pressure of the coat constraining the membrane) should allow it to widen, not to further constrict and break. One could oppose this reasoning in that, at the nanometer scale, close to the size of the membrane, the classic laws of membrane mechanics do not fully apply. But the conductance measurements suggest a widening. So why is the tube still breaking in those experiments? The model used to calculate the radius by conductance relies on the measurement of tube length (very precise in this case, as measured by a micromanipulator with a few $\mathrm{nm}$ precision) and the assumption that the tube is perfectly cylindrical 
(i.e., of same radius everywhere). But if the tube is partially coated, or if there are places where the tube is highly constricted, and other places where the tube has expanded, it is very difficult to estimate the aggregate value of the total conductance. Thus, the increase in conductance may well be a signature of a dramatic change of the tube's shape, with constricted and widened parts, rather than a dynamin depolymerization effect.

But still, approximately $50 \%$ of dynamin dissociates from the membrane when GTP is added $[4,10,33]$. Surprisingly, when looking at the polymerized dynamin on long tubules, no obvious depolymerization occurred when GTP was added $[14,18]$. It might be only the membranebound, non-polymerized (non-tubular) pool of dynamin that comes off in the presence of GTP.

Finally, many experiments correlate fission with disassembly $[23,33]$, but it is indeed very difficult to find the causal link between the two events. It may be that constriction causes fission, depriving the dynamin helix of its substrate, causing it to disassemble. But a point of agreement between the "fission at the edge" and the "depolymerization" model is the requirement for both a coated and an uncoated membrane prior to fission.

\section{Curvature by the $\mathbf{P H}$ domain}

Dynamin was found to bind to liposomes in a curvaturedependent manner [35]. By studying the structure of the PH domain in detail, a hydrophobic loop (VL1) was discovered and shown by elegant fluorescence assays to insert into the outer leaflet of the membrane [35]. Mutation of hydrophobic residues, in particular I533A, reduced membrane fission and transferrin uptake in vivo [36], supporting a role of the $\mathrm{PH}$ domain in the fission reaction. These mutations also dramatically reduced membrane binding and tubulation activities [36], which may account for part of the reduced fission activity of this mutant. It was suggested that the hydrophobic loop induced additional further membrane curvature by a wedge-like mechanism, finalizing constriction by forcing the membrane to curve. In this mechanism, fission is promoted by the destabilization of the membrane and constriction, which does not result from the twisting of the dynamin helix. When used in the conductance/membrane tube assay described above, the I533A mutant also failed to promote fission in the presence of GTP [37]. However, this inhibition of fission was reversed by the addition of more $\mathrm{PIP}_{2}$, suggesting that the deficient fission activity of this mutant could result from a lowered lipidic affinity rather than the lack of membrane insertion.

Of course, both constriction by torsion and the insertion of the PH domain loop can have additive effects, and it is necessary to establish the contribution of each in inducing membrane fission. Amphipathic helices that act as a wedge to sense (e.g., amphipathic lipid packing sensor motif) or create curvature (e.g., BIN/amphiphysin/Rvs [BAR] domain proteins) usually have approximately 15 amino acids [38], much larger than the hydrophobic loop of dynamin that has only seven amino acids. So the structure itself may suggest that the insertion effect is minor. However, the pressure exerted by the constricting helix onto the $\mathrm{PH}$ domain may compensate for this small insertion loop, by forcing it deeper into the lipid leaflet. Thus, the combination of an inserting loop with constriction may act as a "sharpened scissor", whereas constriction alone would not be sharp enough to cut by itself.

The study of the positioning of the $\mathrm{PH}$ domain on the membrane led to another surprising finding. In the constricted tube cryo-EM reconstruction, the PH domain is slightly tilted from the axis perpendicular to the membrane $[9,11]$. Shnyrova et al. proposed that the tilting of the $\mathrm{PH}$ domain, when combined with the insertion of the VL1 loop, would force the membrane to curve in between two rings of dynamin [37]. A mathematical model predicts that this curvature would be sufficient to generate hemi- and full fission. Cryo-EM data and recent studies of the various positions of the PH domain on the membrane, as a result of specific interactions between the membrane and three variable loops of the PH domain [39], support the tilted orientation of the PH domain on the membrane. However, there is no clear experimental indication that the binding and/or the tilting of the $\mathrm{PH}$ domain would create curvature [37], (rather the opposite, see [39]). The authors, from their mathematical model [37], calculated the elastic stress energy generated by tilting and insertion, and compared it to the $\mathrm{PH}$ membrane binding energy, concluding that the PH domain could not come off the membrane in this case. Besides supporting this model, quantitative experimental verification of this prediction would allow the testing of this model in more constricted conformations [16], in which the $\mathrm{PH}$ domain may be even more tilted, in this case, breaking its bond to the membrane.

In conclusion, the fact that constriction alone is not sufficient for fission and that membrane tension is also required can be explained by the observation of fission occurring at the edge of the dynamin helix. The tilting of the PH domain and its insertion loop may "sharpen the knife" of dynamin by further destabilizing the membrane under constriction, but the exact contribution of these effects is yet to be quantified. Trying to reconcile all the data, one could suggest the following model. Dynamin rings would constrict under GTP hydrolysis to a radius close to hemi-fission. Elastic stress in the 
membrane at the edge of dynamin would then help to finalize the break. Because of the constriction, the PH domain would insert into the outer leaflet, further promoting fission. Constriction would also tilt the $\mathrm{PH}$ domains, causing the links to $\mathrm{PIP}_{2}$ to break. Once fission occurred, and because the bonds to the membrane are broken, the membrane retracts itself from the dynamin helix, causing it to disassemble.

\section{What is the future of the dynamin field?}

After 25 years of work on dynamin, an extensive knowledge of the various aspects of its biological function has been acquired. The molecule itself, because of its unusual structure and function, has stimulated the development of innovative assays both in vitro and in vivo. But there is more to understand, in particular the regulation of the GTP cycle and the precise function of dynamin-related proteins. In the following section, I suggest possible research directions for the future.

\section{Coupling of the GTP cycle to dynamin constriction}

Even though we have found conditions in which dynamin can mediate membrane fission in reconstituted assays, and we now have a detailed understanding of the structure and of the conformational changes of dynamin, we have very little information on how the conformational change is coupled to the GTP hydrolysis cycle. One of the main reasons for this is the lack of crystal structures of full-length dynamin in the presence of nucleotides. However, there is one exception: the GG construct [40]—a part of dynamin that contains the GTPase domain, a hinge (or bundle signaling element [BSE]), and a small piece of the rigid stalk—has been crystallized [41] with GMPPCP and GDP. $\mathrm{AlF}^{4-}$. These structures clearly show a conformational change in the dynamin-GDP.AlF ${ }^{4-}$ structure in which there is a dramatic rotation of the BSE around the GTPase domain totally changing the relative orientation of the BSE and the GTPase domain. However, the GG structure in the presence of GDP.AlF ${ }^{4-}$ could not be docked in any of the 3D maps of the dynamin helix [9], indicating that this structure is either not representative of the full-length structure, or it represents an intermediate state of dynamin that is not observed in the cryo-EM data.

One possible future direction of the dynamin field is to unravel the different structures of dynamin at the dimeric level as well as at the helical structural level with various analogs of GTP, in order to gain insights into how the powerstroke [9] observed at the structural level is generated by the GTP hydrolysis.

Two recent findings will further feed into the discussion on the regulation of the dynamin GTPase cycle. Among the mechanoenzymes, dynamin is one of the very few to use GTP instead of ATP. Intracellular GTP concentration is lower than ATP, which may make it a less efficient source of energy for a motor protein. The nucleotide-diphosphate kinase (NDPK) nm-23 was found to interact directly with dynamin to locally fuel dynamin with GTP [42]. The authors concluded that the very high enzymatic activity of NDPKs can locally increase the GTP concentration to enhance the power of dynamin mechanics. The NDPKdynamin interaction could also represent another level of regulation of the GTPase cycle.

Also, there are two studies using fluorescence to measure the number of dynamin molecules present at the neck of CCPs (non-abortive events only) by quantitative total internal reflection fluorescence imaging [43], or spinning-disk (Cocucci et al., unpublished data). Most endocytic events have between 26 and 60 molecules, representing one or two rings of dynamin. From the crystal structure, and helical reconstruction, it is expected that the GTPase activity would be most efficient in an organization of at least two rings, as the GTPase domain can interact from turn-to-turn to increase the GTPase activity [13]. In vitro support of the need for two rings came from the conductance assay described above [37]. So the fact that efficient fission can be mediated by less than two rings in vivo is a surprise and will obviously trigger further intensive studies to resolve this discrepancy. There are at least two explanations for the difference between in vitro and in vivo data. Firstly, dynamin in vivo may reach optimal GTPase activity through its interaction with NDPKs, and therefore does not require the two-ring state to be reached. Secondly, the dynamin helix probably polymerizes with other partners (such as endophilin and amphiphysin) that, together, form a helical scaffold. This BAR domain scaffold may provide a solid anchoring point for efficient constriction of a single ring of dynamin. However, the regulation of the dynamin GTPase activity by BAR domains is still a matter of intense debate [44] and some reports $[45,46]$ actually found a strong inhibition of the GTPase domain by BAR proteins. Moreover, the fact than dynamin K44A assembles into a double helix in the presence of GTP [16] may offer a solution to the apparent discrepancy between structural and cell biology data: in the presence of GTP, it is possible that the dynamin assembles directly into a constricted state, in a two-star helix. Constriction could thus be realized by a single dynamin ring, made of two facing filaments in a double helix [16].

Regardless of these controversies, the study of the fission reaction mediated by dynamin in vivo will provide additional surprising insight and allow us to understand how the GTPase cycle is regulated in cells. 


\section{Roles of the dynamin partners: actin and membrane remodeling proteins}

In clathrin-mediated endocytosis, dynamin does not function alone to trigger membrane fission: through its proline-rich domain (PRD), it binds to many partners [47]. The generally accepted roles of these partners are to regulate and to orchestrate the sequence of events leading to fission in vivo [48]. In particular, the family of BAR domain-containing proteins has been shown to play various roles in clathrin-mediated endocytosis [44]. Among them, three roles are still intensively debated. Firstly, it has been suggested that the crescent-shaped BAR domain binds the membrane in a curvature-sensing manner [49], spontaneously localizing to the highly curved necks of CCPs [34,50]. Then, these BAR domain proteins can recruit dynamin to the neck through their SH3 domain [51]. The second role of BAR proteins could be participation in generating membrane curvature $[45,52]$, as these proteins can tubulate liposomes in vitro. The balance between the curvature-inducing and curvature-sensing abilities of BAR proteins is dependent upon the membrane-bound density of the protein [53]. But as it is rather difficult to estimate in vivo densities, the precise in vivo role of the BAR domains is unclear. The third role of BAR proteins, which has been under intensive study in recent years, is the regulation of clathrin-mediated endocytosis. In particular, endophilin has been implicated in the recruitment of synaptojanin [29], a phosphatase important for the regulation of phosphoinositides at CCPs and that plays a major role in controlling dynamin-mediated fission [31]. F-BAR proteins, a subclass of the BAR proteins with smaller intrinsic curvature, are critical for the recruitment of actin to the CCPs [54]. As discussed above, actin is a major player in dynamin-mediated fission because it generates the necessary tension required for fission. Thus, even though the large number of BAR domain family proteins all share common structural features, they have numerous and diverse roles in clathrin-mediated endocytosis.

\section{Conclusion}

In conclusion, dynamin, because of its unique structural features and function, has been under intense scrutiny for the past 25 years. The original model proposed for its function, the constriction model $[3,50]$, has gained strong support from experimental observations. We now have a better understanding of how it constricts, and what the sequence of events leading to fission could be, if fission is mediated by constriction (see Figure 1 and the youtube video: https://www.youtube.com/watch? $\mathrm{v}=\mathrm{q}-\mathrm{Er} 5 \mathrm{sEaj} 2 \mathrm{U}$ [Accessed 19 September 2014]). All other models proposed (see [1] for review) have less experimental support compared to the constriction model. However, many aspects of dynamin function
Figure I. Putative constriction cycle of the dynamin helix

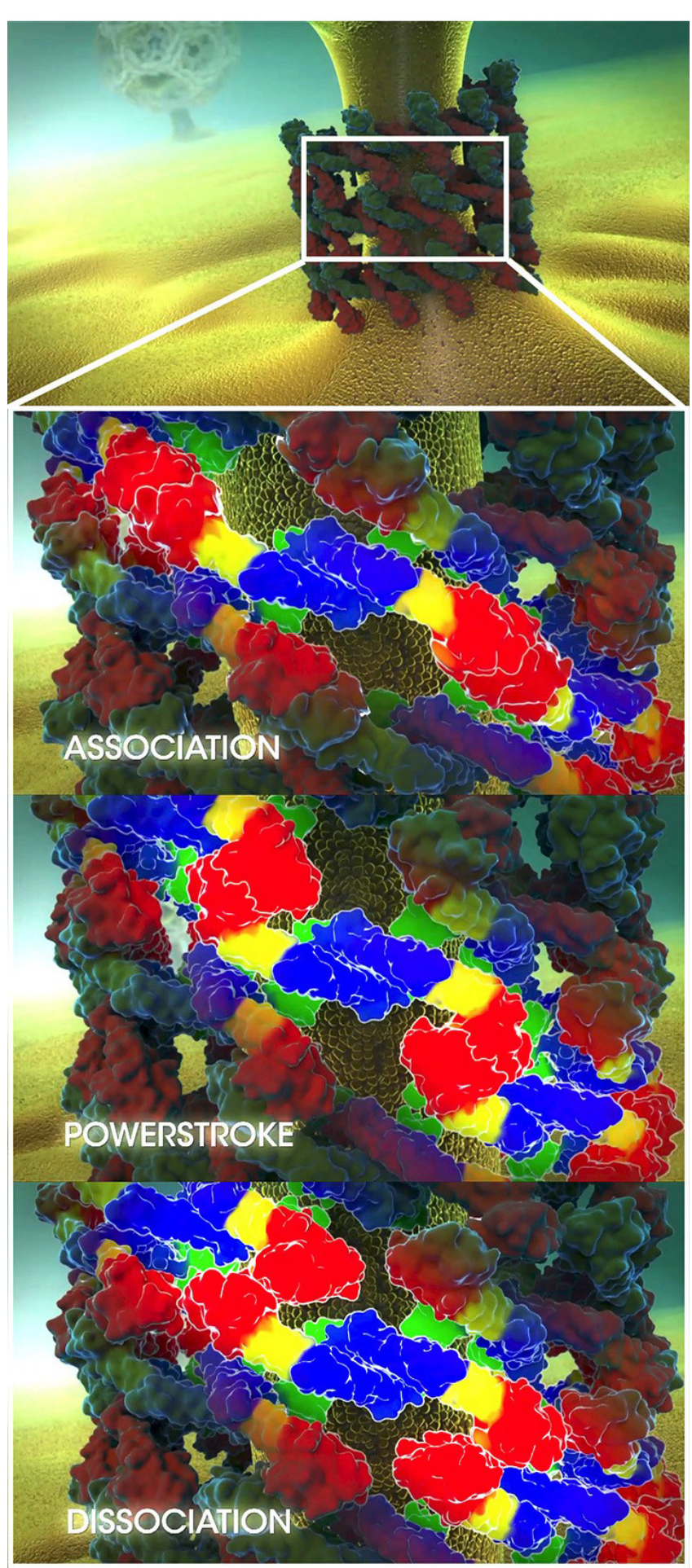

Constriction of dynamin is thought to happen by the twisting of the helix through several cycles of association/powerstroke/dissociation of GTPase domains (red) of the adjacent turns of dynamin. See the animated version at: https://www.youtube.com/watch?v=q-Er5sEaj2U (Accessed 19 September 2014). 
are still unclear. Firstly, the exact coupling between GTPase activity and the conformational change are poorly characterized. Secondly, the necessarily tight regulation of dynamin function requires many partners whose roles are still far from being understood. Finally, dynamin is part of a wide family of proteins involved in various fission and fusion reactions [55]. Further confirmation of the constriction model will probably come from the in-depth study of these other fission molecules.

\section{Abbreviations}

3D, three-dimensional; BAR, BIN/amphiphysin/Rvs; BSE, bundle signaling element; CCP, clathrin-coated pit; cryo-EM, cryo-electron microscopy; NDPK, nucleotidediphosphate kinase; $\mathrm{PIP}_{2}$, phosphatidylinositol 4,5bisphosphate; $\mathrm{PH}$, pleckstrin homology.

\section{Disclosures}

The author declares that he has no disclosures.

\section{Acknowledgements}

I would like to thank Cameron Scott for his corrections and discussion about this manuscript, as well as Sandy Schmid and Jenny Hinshaw for their passionate discussions about dynamin and this article.

\section{References}

I. Morlot S, Roux A: Mechanics of dynamin-mediated membrane fission. Annu Rev Biophys 2013, 42:629-49.

2. Ford Marijn GJ, Jenni S, Nunnari J: The crystal structure of dynamin. Nature 20II, 477:56I-6.

\section{FlOOOPrime} RECOMMENDED

3. Hinshaw JE, Schmid SL: Dynamin self-assembles into rings suggesting a mechanism for coated vesicle budding. Nature 1995, 374:190-2.

4. Warnock DE, Hinshaw JE, Schmid SL: Dynamin self-assembly stimulates its GTPase activity. J Biol Chem 1996, 27 I:223 10-4.

5. Sweitzer SM, Hinshaw JE: Dynamin undergoes a GTP-dependent conformational change causing vesiculation. Cell 1998, 93: $1021-9$.

6. Takei K, Haucke V, Slepnev V, Farsad K, Salazar M, Chen H, Camilli $P$ de: Generation of coated intermediates of clathrinmediated endocytosis on protein-free liposomes. Cell I998, 94: $|3|-4 \mid$.

7. Roux A, Koster G, Lenz M, Sorre B, Manneville J, Nassoy P, Bassereau P: Membrane curvature controls dynamin polymerization. Proc Natl Acad Sci USA 2010, 107:4141-6.

8. Chen $\mathrm{Y}$, Zhang $\mathrm{P}$, Egelman $\mathrm{EH}$, Hinshaw JE: The stalk region of dynamin drives the constriction of dynamin tubes. Nat Struct Mol Biol 2004, I I:574-5.

\section{FlOOOPrime}

RECOMMENDED

9. Chappie JS, Mears JA, Fang S, Leonard M, Schmid SL, Milligan RA, Hinshaw JE, Dyda F: A pseudoatomic model of the dynamin polymer identifies a hydrolysis-dependent powerstroke. Cell 20II, | 47:209-22.

FIOOOPrime
RECOMMENDED

10. Danino D, Moon K, Hinshaw JE: Rapid constriction of lipid bilayers by the mechanochemical enzyme dynamin. J Struct Biol 2004, I 47:259-67.

II. Mears JA, Ray P, Hinshaw JE: A corkscrew model for dynamin constriction. Structure 2007, I 5: I | 90-202.

12. Faelber K, Posor Y, Gao S, Held M, Roske Y, Schulze D, Haucke V, Noé F, Daumke O: Crystal structure of nucleotide-free dynamin. Nature 201 I, 477:556-60.

\section{FlOOOPrime
RECOMMENDED}

13. Faelber K, Held M, Gao S, Posor Y, Haucke V, Noé F, Daumke O: Structural insights into dynamin-mediated membrane fission. Structure 2012, 20:1621-8.

\section{FlOOOPrime}

RECOMMENDED

14. Roux A, Uyhazi K, Frost A, Camilli P de: GTP-dependent twisting of dynamin implicates constriction and tension in membrane fission. Nature 2006, 441:528-31.

FlOOOPrime
RECOMMENDED

15. Zhang $P$, Hinshaw JE: Three-dimensional reconstruction of dynamin in the constricted state. Nat Cell Biol 200I, 3:922-6.

16. Sundborger AC, Fang S, Heymann JA, Ray P, Chappie JS, Hinshaw JE: A Dynamin Mutant Defines a Superconstricted Prefission State. Cell Reports 2014.

\section{FlOOOPrime}

RECOMMENDED

17. Kozlovsky Y, Kozlov MM: Membrane fission: model for intermediate structures. Biophys J 2003, 85:85-96.

18. Morlot S, Galli V, Klein M, Chiaruttini N, Manzi J, Humbert F, Dinis L, Lenz M, Cappello G, Roux A: Membrane shape at the edge of the dynamin helix sets location and duration of the fission reaction. Cell 2012, I5 I:619-29.

FlOOOPrime RECOMMENDED

19. Itoh T, Erdmann KS, Roux A, Habermann B, Werner H, Camilli P de: Dynamin and the actin cytoskeleton cooperatively regulate plasma membrane invagination by BAR and F-BAR proteins. Dev Cell 2005, 9:79I-804.

\section{FlOOOPrime}

\section{RECOMMENDED}

20. Kaksonen M, Toret CP, Drubin DG: A modular design for the clathrin- and actin-mediated endocytosis machinery. Cell 2005, 1 23:305-20.

\section{FlOOOPrime}

\section{RECOMMENDED}

21. Liu J, Sun Y, Oster GF, Drubin DG: Mechanochemical crosstalk during endocytic vesicle formation. Curr Opin Cell Biol 20 I 0, 22:36-43.

22. Merrifield C], Perrais D, Zenisek D: Coupling between clathrincoated-pit invagination, cortactin recruitment, and membrane scission observed in live cells. Cell 2005, I 2 I:593-606.

\section{FlOOOPrime}

RECOMMENDED

23. Bashkirov PV, Akimov SA, Evseev AI, Schmid SL, Zimmerberg J, Frolov VA: GTPase cycle of dynamin is coupled to membrane squeeze and release, leading to spontaneous fission. Cell 2008, I35: 1276-86.

FlOOOPrime RECOMMENDED

24. Pinot M, Vanni S, Pagnotta S, Lacas-Gervais S, Payet L, Ferreira T, Gautier R, Goud B, Antonny B, Barelli H: Lipid cell biology. 
Polyunsaturated phospholipids facilitate membrane deformation and fission by endocytic proteins. Science 20I4, 345:693-7.

\section{FlOOOPrime}

25. Watanabe S, Rost BR, Camacho-Pérez M, Davis MW, SöhlKielczynski B, Rosenmund C, Jorgensen EM: Ultrafast endocytosis at mouse hippocampal synapses. Nature 2013, 504:242-7.

\section{FlOOOPrime}

26. Watanabe S, Liu Q, Davis MW, Hollopeter G, Thomas N, Jorgensen NB, Jorgensen EM: Ultrafast endocytosis at Caenorhabditis elegans neuromuscular junctions. Elife 2013, 2:e00723.

\section{FlOOOPrime} RECOMMENDE

27. McPherson PS, Garcia EP, Slepnev VI, David C, Zhang X, Grabs D, Sossin WS, Bauerfeind R, Nemoto Y, Camilli P de: A presynaptic inositol-5-phosphatase. Nature 1996, 379:353-7.

\section{FlOOOPrime}

RECOMMENDED

28. Cremona O, Di Paolo G, Wenk MR, Lüthi A, Kim WT, Takei K, Daniell L, Nemoto Y, Shears SB, Flavell RA, McCormick DA, Camilli $P$ de: Essential role of phosphoinositide metabolism in synaptic vesicle recycling. Cell 1999, 99:179-88.

\section{FlOOOPrime \\ RECOMMENDED}

29. Perera RM, Zoncu R, Lucast L, Camilli P de, Toomre D: Two synaptojanin $I$ isoforms are recruited to clathrin-coated pits at different stages. Proc Natl Acad Sci USA 2006, 103:19332-7.

\section{FlOOOPrime}

\section{RECOMMENDED}

30. Hayashi M, Raimondi A, O'Toole E, Paradise S, Collesi C, Cremona O, Ferguson SM, Camilli $P$ de: Cell- and stimulus-dependent heterogeneity of synaptic vesicle endocytic recycling mechanisms revealed by studies of dynamin I-null neurons. Proc Natl Acad Sci USA 2008, 105:2175-80.

\section{FlOOOPrime}

\section{RECOMMENDED}

31. Chang-lleto B, Frere SG, Chan RB, Voronov SV, Roux A, Di Paolo G: Synaptojanin I-mediated $\mathrm{PI}(4,5) \mathrm{P} 2$ hydrolysis is modulated by membrane curvature and facilitates membrane fission. Dev Cell 20II, 20:206-18.

FlOOOPrime RECOMMENDED

32. Milosevic I, Giovedi S, Lou X, Raimondi A, Collesi C, Shen H, Paradise S, O'Toole E, Ferguson S, Cremona O, Camilli $P$ de: Recruitment of endophilin to clathrin-coated pit necks is required for efficient vesicle uncoating after fission. Neuron 20II, 72:587-60I.

\section{FlOOOPrime}

RECOMMENDE

33. Pucadyil TJ, Schmid SL: Real-time visualization of dynamin-catalyzed membrane fission and vesicle release. Cell 2008, 135: |263-75.

\section{FlOOOPrime \\ RECOMMENDED}

34. Merrifield CJ, Feldman ME, Wan L, Almers W: Imaging actin and dynamin recruitment during invagination of single clathrincoated pits. Nat Cell Biol 2002, 4:691-8.

\section{FlOOOPrime \\ RECOMMENDED}

35. Ramachandran R, Schmid SL: Real-time detection reveals that effectors couple dynamin's GTP-dependent conformational changes to the membrane. EMBO / 2008, 27:27-37.

\section{FlOOOPrime}

\section{RECOMMENDED}

36. Ramachandran R, Pucadyil TJ, Liu Y, Acharya S, Leonard M, Lukiyanchuk V, Schmid SL: Membrane insertion of the pleckstrin homology domain variable loop I is critical for dynamin-catalyzed vesicle scission. Mol Biol Cell 2009, 20:4630-9.

FlOOOPrime

37. Shnyrova AV, Bashkirov PV, Akimov SA, Pucadyil TJ, Zimmerberg J, Schmid SL, Frolov VA: Geometric catalysis of membrane fission driven by flexible dynamin rings. Science 2013, 339:1433-6.

\section{FlOOOPrime}

\section{RECOMMENDED}

38. Antonny B: Mechanisms of membrane curvature sensing. Annu Rev Biochem 20II, 80:101-23.

39. Mehrotra N, Nichols J, Ramachandran R: Alternate pleckstrin homology domain orientations regulate dynamin-catalyzed membrane fission. Mol Biol Cell 2014, 25:879-90.

\section{FlOOOPrime}

\section{RECOMMENDED}

40. Chappie JS, Acharya S, Liu Y, Leonard M, Pucadyil TJ, Schmid SL: An intramolecular signaling element that modulates dynamin function in vitro and in vivo. Mol Biol Cell 2009, 20:356I-7I.

\section{FlOOOPrime \\ RECOMMENDED}

4I. Chappie JS, Acharya S, Leonard M, Schmid SL, Dyda F: G domain dimerization controls dynamin's assembly-stimulated GTPase activity. Nature 2010, 465:435-40.

\section{FlOOOPrime RECOMMENDED}

42. Boissan M, Montagnac G, Shen Q, Griparic L, Guitton J, Romao M Sauvonnet N, Lagache T, Lascu I, Raposo G, Desbourdes C, Schlattner U, Lacombe M, Polo S, van der Bliek, Alexander M, Roux A, Chavrier P: Membrane trafficking. Nucleoside diphosphate kinases fuel dynamin superfamily proteins with GTP for membrane remodeling. Science 20I4, 344:15I0-5.

43. Grassart A, Cheng AT, Hong SH, Zhang F, Zenzer N, Feng Y, Briner DM, Davis GD, Malkov D, Drubin DG: Actin and dynamin2 dynamics and interplay during clathrin-mediated endocytosis. J Cell Biol 20I4, 205:72I-35.

\section{FlOOOPrime}

\section{RECOMMENDED}

44. Daumke O, Roux A, Haucke V: BAR domain scaffolds in dynamin-mediated membrane fission. Cell 2014, 156:882-92.

\section{FlOOOPrime} RECOMMENDED

45. Farsad K, Ringstad N, Takei K, Floyd SR, Rose K, Camilli P de: Generation of high curvature membranes mediated by direct endophilin bilayer interactions. J Cell Biol 200I, I55:193-200.

46. Neumann S, Schmid SL: Dual role of BAR domain-containing proteins in regulating vesicle release catalyzed by the GTPase, dynamin-2. J Biol Chem 2013, 288:25I 19-28.

\section{FlOOOPrime}

47. Slepnev VI, Camilli P de: Accessory factors in clathrin-dependent synaptic vesicle endocytosis. Nat Rev Neurosci 2000, I:16I-72.

48. Taylor MJ, Perrais D, Merrifield C]: A high precision survey of the molecular dynamics of mammalian clathrin-mediated endocytosis. PLoS Biol 20II, 9:el000604.

\section{FlOOOPrime}

\section{RECOMMENDED}

49. Peter BJ, Kent HM, Mills IG, Vallis Y, Butler P, Jonathan G, Evans PR, McMahon HT: BAR domains as sensors of membrane curvature: the amphiphysin BAR structure. Science 2004, 303:495-9.

\section{FlOOOPrime} RECOMMENDED

50. Takei K, McPherson PS, Schmid SL, Camilli P de: Tubular membrane invaginations coated by dynamin rings are induced by GTP. gamma S in nerve terminals. Nature 1995, 374:186-90. 
51. Shupliakov O, Löw P, Grabs D, Gad H, Chen H, David C, Takei K, Camilli P de, Brodin L: Synaptic vesicle endocytosis impaired by disruption of dynamin-SH3 domain interactions. Science 1997, 276:259-63.

52. Takei K, Slepnev VI, Haucke V, Camilli P de: Functional partnership between amphiphysin and dynamin in clathrin-mediated endocytosis. Nat Cell Biol 1999, I:33-9.

53. Sorre B, Callan-Jones A, Manzi J, Goud B, Prost J, Bassereau P, Roux $A$ : Nature of curvature coupling of amphiphysin with membranes depends on its bound density. Proc Natl Acad Sci USA 2012, 109:173-8.

54. Frost A, Unger VM, Camilli P de: The BAR domain superfamily: membrane-molding macromolecules. Cell 2009, I37:191-6.

FlOOOPrime

RECOMMENDED

55. Praefcke Gerrit JK, McMahon HT: The dynamin superfamily: universal membrane tubulation and fission molecules? Nat Rev Mol Cell Biol 2004, 5:I33-47. 